\title{
Identification of TCIRG1 and CLCN7 gene mutations in a patient with autosomal recessive osteopetrosis
}

\author{
TINGTING YU ${ }^{1,2^{*}}$, YONGGUO YU ${ }^{2,3,4 *}$, JIAN WANG ${ }^{5 *}$, LEI YIN ${ }^{3,4}$, YUNFANG ZHOU $^{4}$, DAMING YING ${ }^{4}$, \\ RONGKUI HUANG ${ }^{4}$, HUIJIN CHEN $^{4}$, SHENMEI WU ${ }^{4}$, YONGNIAN SHEN ${ }^{3,4}$, QIHUA FU $^{5}$ and FUXIANG CHEN ${ }^{1}$ \\ ${ }^{1}$ Department of Laboratory Medicine, Shanghai Ninth People's Hospital, Shanghai Jiaotong University School of Medicine, \\ Shanghai 200011; ${ }^{2}$ Institute of Pediatric Translational Medicine; ${ }^{3}$ Department of Internal Medicine; \\ ${ }^{4}$ Rare Diseases Outpatient Clinic; ${ }^{5}$ Department of Laboratory Medicine, Shanghai Children's Medical Center, \\ Shanghai Jiaotong University School of Medicine, Shanghai 200127, P.R. China
}

Received June 27, 2013; Accepted February 4, 2014

DOI: $10.3892 / \mathrm{mmr} .2014 .1955$

\begin{abstract}
Osteopetrosis is a heritable bone disorder that exhibits highly clinical and genetical heterogeneity, and is caused by defective osteoclastic resorption. The three main forms are the autosomal recessive severe (ARO), the intermediate autosomal and the autosomal dominant benign osteopetrosis forms. In the present study, the clinical, biochemical and radiological manifestations were described in a patient with osteopetrosis. Sequence analysis identified the compound heterozygous mutations, c.909C >A (p.Tyr303X) and c.2008C >T (p.Arg670X), in TCIRG1, and a heterozygous splicing mutation, c.1798-1G>T, in the chloride channel 7 gene (CLCN7). Two aberrant forms of the CLCN7 transcripts, c.1798_1883 (exon 20) deletion predicted to cause p.Leu601GlyfsX13, and the c.1798_1821 deletion, the first $24 \mathrm{bp}$ of the exon 20, predicted to cause p.Gly600_Gln607del, were detected by further analysis of the splicing patterns in the leukocytes. The patient's asymptomatic mother carried the TCIRG1 c.909C >A (p.Tyr303X) and $C L C N 7 \mathrm{c} .1798-1 \mathrm{G}>\mathrm{T}$ mutations, while the asymptomatic father carried the TCIRG1 c.2008C $>\mathrm{T}$ (p.Arg670X) mutation only. The patient was finally diagnosed with ARO on the basis of clinical and biochemical parameters, radiological changes and genetic defects. To the best of our knowledge, this is the first reported case of a patient with osteopetrosis who carries
\end{abstract}

Correspondence to: Dr Qihua Fu, Department of Laboratory Medicine, Shanghai Children's Medical Center, Shanghai Jiaotong University School of Medicine, 1,678 Dongfang Road, Shanghai 200127, P.R. China

E-mail: qfu@shsmu.edu.cn

Dr Fuxiang Chen, Department of Laboratory Medicine, Shanghai Ninth People's Hospital, Shanghai Jiaotong University School of Medicine, 639 Zhizaoju Road, Shanghai 200011, P.R. China

E-mail: chenfx@sjtu.edu.cn

*Contributed equally

Key words: osteopetrosis, TCIRG1, CLCN7, mutation, phenotype
TCIRG1 and CLCN7 mutations. In addition, among the three mutations, TCIRG1 c.909C >A and CLCN7 c.1798-1G>T were novel mutations.

\section{Introduction}

Bone development and homeostasis is achieved by a strict balance between bone formation by osteoblasts and resorption by osteoclasts. Defective bone resorption results in osteopetrosis, an inherited disorder encompassing a clinical and genetical heterogenous group of conditions. Three main forms are classified on the basis of inheritance patterns, age of onset and severity. These are the autosomal recessive severe (ARO; MIM 259700), intermediate autosomal (IAO) and autosomal dominant benign osteopetrosis (ADO) forms (1). All these forms are characterized by an increased bone density, which may result in various phenotypical features, including fractures, osteomyelitis, deformity, dental abnormalities, bone marrow impairment and cranial nerve compression. (2). Osteopetrosis is a rare condition, and the estimated incidence of ARO is 1 in 250,000 births, while that of ADO is 1 in 20,000 births $(3,4)$.

Mutations in at least 10 genes have been identified as causative in various types of osteopetrosis cases in humans (2). However, the majority of the osteopetrosis cases reported thus far are caused by defects in gene products involved in the regulation of the intra- and extracellular $\mathrm{pH}$ of osteoclasts. Two significant molecules that are involved in the acidification machinery are the proton pump, vacuolar ATPase (V-ATPase), and the chloride-specific ion channel, CLCN7. Defects in TCIRG1, which encodes the V-ATPase a3 subunit, have been reported to be responsible for ARO in $>50 \%$ of patients (5-7). However, mutations in the $C L C N 7$ gene give rise to the complete spectrum of osteopetrosis, underlying $15 \%$ of all ARO cases, almost all known cases of IAO and 75\% of ADO type II cases (ADO II; Albers-Schönberg disease; MIM 166600) (8-10).

\section{Materials and methods}

Patient. A 21-month-old male was admitted to Shanghai Children's Medical Center (Shanghai, China) due to persistent 
anemia and a lack of dentition. The patient was full-term at birth, with a normal weight and length, and a subsequent weight and length of $12 \mathrm{~kg}$ (50th percentile) and $84.8 \mathrm{~cm}$ (between 25th and 50th percentile), respectively, at 21 months. A prominent forehead, enlarged abdomen with moderate hepatosplenomegaly and visual disturbance were noted during the physical examination. Laboratory tests revealed that the patient had moderate anemia, elevated levels of serum alkaline phosphatase, parathyroid hormone, creatine kinase and $\mathrm{MB}$ isoenzyme, and decreased serum $\mathrm{Ca}^{2+}$ levels. The levels of phosphonium, 1,25-dihydroxy vitamin D3, lactate dehydrogenase, thyroid hormone and thyroid stimulating hormone were within the normal ranges. Imaging examinations consisting of computerized tomography (CT) scans (LightSpeed 16 Slice CT; GE Healthcare, Fairfield, CT, USA) and X-rays (AMX IV Plus Portable X-Ray, GE Healthcare) revealed a general increase of bone density involving the skull, vertebrae and limbs (Fig. 1). The diagnosis of osteopetrosis was based on the skeletal radiographs along with the clinical and laboratory data. The patient was scheduled to undergo a bone marrow transplant; however, died of an infection following intensive chemotherapy. The patient was the only son of a non-related couple. No clinical abnormalities were noted in the parents, and further biochemical and radiological examinations of the patient's mother also appeared normal.

Molecular analysis. Ethylenediaminetetraacetic acid-peripheral blood samples were obtained from the patient, his parents and 100 healthy individuals. Genomic DNA was isolated from the peripheral blood leukocytes using the QIAmp DNA Blood kit (Qiagen, Hilden, Germany). All the exons and exon-intron boundaries of the TCIRG1 and CLCN7 genes from the patient's genomic DNA were amplified by PCR, and the primer sequences are listed in Tables I and II. The PCR products were analyzed by direct DNA sequencing on an ABI 3700 sequencer (Applied Biosystems, Foster City, CA, USA). Only genomic fragments containing the mutations identified in the patient were amplified and sequenced for the parents and the normal controls. Several polymorphisms, including single-nucleotide polymorphisms (SNPs), rs12926089, rs12926669 and rs960467, and the variable number tandem repeat (VNTR) in intron 8 of $C L C N 7$ were also analyzed in the pedigree.

Reverse transcription-polymerase chain reaction ( $R T-P C R)$. The total RNA of the patient and the parents was isolated from their peripheral leukocytes using the QIAmp RNA Blood kit (Qiagen). The first-strand cDNA was synthesized using random primers, oligo primers and PrimeScript Reverse Transcriptase (Takara Biotechnology (Dalian) Co., Ltd., Dalian, China). The synthesized products were amplified with primer P1, 5'-TACGGGCTCACGGTGTCTG-3', which is located in exon 17, and primer P2, 5'-GGTAGGCGTCTCGGAAGTC-3', which is located in exon 23 of CLCN7. The PCR products were further amplified using semi-nested oligonucleotide primers P1 and P3, 5'-TTGTGCTTTAGGAGAACGA-3', which is located in exon 22 of $C L C N 7$. The products were cloned into the pMD 18-T vector (Takara Biotechnology (Dalian) Co., Ltd.) and 30 clones were selected and sequenced.
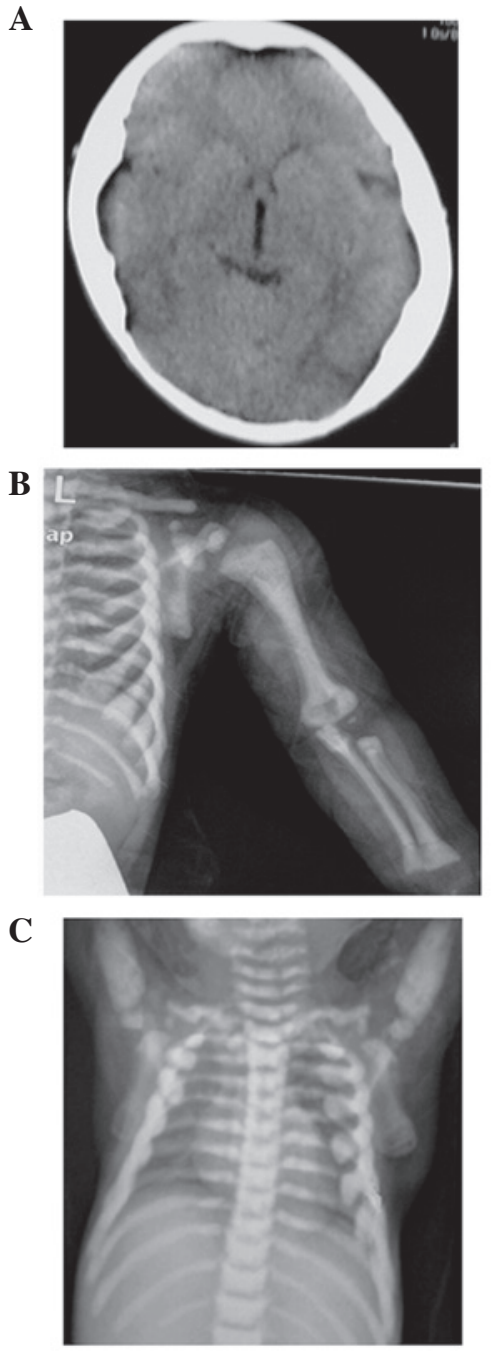

Figure 1. Imaging examinations consisting of a CT scan and X-rays of the proband. A generalized increase in bone density involving (A) the skull, (B) the limbs and (C) the vertebrae was observed. CT, computed tomography.

Ethics statement. The study was approved by the Ethics Committee of Shanghai Children's Medical Center, Shanghai Jiao Tong University School of Medicine. Written informed consent was obtained from the parents and the 100 healthy individuals prior to blood sampling and DNA analysis.

\section{Results}

Molecular analysis of the TCIRG1 and CLCN7 genes. Direct genomic DNA sequencing of the patient revealed compound heterozygous mutations, c.909C>A (p.Tyr303X) and c.2008C > T (p.Arg670X), in TCIRG1 and a heterozygous splice site mutation, c.1798-1G>T, in the CLCN7 gene (Fig. 2). The patient's mother carried the TCIRG1 c.909C $>$ A (p.Tyr303X) and $C L C N 7$ c.1798-1G $>$ T mutations, while the patient's father carried the TCIRG1 c. $2008 \mathrm{C}>\mathrm{T}$ (p.Arg670X) mutation. The CLCN7 c.1798-1G>T variation was not detected in the 100 healthy individuals. In addition, several SNPs, including rs12926089, rs12926669 and rs960467, and the intron 8 VNTR in the $C L C N 7$ gene were investigated. However, no differences in the investigated polymorphisms were identified between the patient and his mother (Table III). 
Table I. PCR primers used for amplification of TCIRG1 gene.

\begin{tabular}{|c|c|c|}
\hline Primer & Sequence (5' to $\left.3^{\prime}\right)$ & Size (bp) \\
\hline EXON1 F & TCAACСТСТССCAGACTTCC & 320 \\
\hline EXON1 R & CTGAGCTGCATTCACGGAG & \\
\hline EXON2 F & TCAGTGAGTGAAGGTGCACAG & 319 \\
\hline EXON2 R & GTTCAAATGGGGCCAGG & \\
\hline EXON3 F & TCCACACCTTTCTGGAGGAG & 266 \\
\hline EXON3 R & TTTCAGATCAAACTTGGCCC & \\
\hline EXON4+EXON5 F & GAGTTTGGGGCAGCAGG & 564 \\
\hline EXON4+EXON5 R & CACTGGACAAGGAGTCGGAG & \\
\hline EXON6+EXON7 F & GAGGCCTCCTGCCTTCC & 561 \\
\hline EXON6+EXON7 R & GGCCAGAAGGACACAGCTAC & \\
\hline EXON8 F & CCTATCGTGACTCCTCCCC & 262 \\
\hline EXON8 R & АССТССТGCACССАССТС & \\
\hline EXON9 F & GAGGTGGGTGCAGGAGG & 372 \\
\hline EXON9 R & CTGGAAGTGAGGCAGAAACG & \\
\hline EXON10 F & ATCTCCAGCTGGGCCTG & 301 \\
\hline EXON10 R & CCTCAGGCTCACACCCAC & \\
\hline EXON11+EXON12 F & AAGTGATGGGTTCTTGACTGC & 570 \\
\hline EXON11+EXON12 R & AGGAATGCATCACTGCGG & \\
\hline EXON13 F & AGTCTGGCTGGAGGTGAGG & 291 \\
\hline EXON13 R & CACACAGGAGTGCTCAGCG & \\
\hline EXON14+EXON15 F & GGGAAAACAGGGTGGTGAG & 597 \\
\hline EXON14+EXON15 R & GATCTTGCAGCTCCCAGTG & \\
\hline EXON16+EXON17 F & CGTGACTGCTGTGACTCAGG & 604 \\
\hline EXON16+EXON17 R & GCAGAACTCGATGGTGTGG & \\
\hline EXON18 F & GCCTGGATGATGAAGAGGAG & 307 \\
\hline EXON18 R & AACTGAGGCCCAGAGAGAAG & \\
\hline EXON19+EXON20 F & AAGTGGGACTGTCCAAGGAG & 613 \\
\hline EXON19+EXON20 R & TCCCAGATCCTACACCATGC & \\
\hline
\end{tabular}

PCR, polymerase chain reaction; F, forward; $\mathrm{R}$, reverse.

Transcription experiment of the CLCN7 c.1798-1G>T mutation. RT-PCR performed on the RNA extracted from the father enabled the detection of one band of the expected $560 \mathrm{bp}$ (from exon 17 to exon 22 of the CLCN7 gene; Fig. 3A, lane 1). However, the same amplification conducted using RNA extracted from the mother and the patient, resulted in two different PCR fragments (Fig. 3A; lanes 2 and 3). In the patient, cloning and sequencing revealed the presence of the normal transcript corresponding to the 560-bp fragment and of two aberrant forms of the transcripts. The majority of transcripts resulted from the entire deletion of exon 20 (c.1798_1883del; Fig. 3B), which was detected in 11 out of the 30 clones selected. The minority of transcripts resulted from the deletion of the first 24 bp of exon 20 (c.1798_1821del; Fig. 3C), which was detected in three out of the 30 clones picked. Similar deletions were also identified in the patient's mother, while no aberrant transcripts were detected in the father.

\section{Discussion}

In the present study, the case of a patient with osteopetrosis was investigated. Molecular analysis of the patient revealed compound heterozygous nonsense mutations in TCIRG1 and a heterozygous splice site mutation in CLCN7. Among the three mutations, TCIRG1 c.909C >A (p.Tyr303X) and CLCN7c.1798-1G>T were novel mutations. CLCN7 encodes the chloride-specific ion channel, CLCN7, which cooperates with the gene product of TCIRG1, the a3 subunit of V-ATPase (11). CLCN7 is essential for efficient proton pumping due to its role in neutralizing current, and is involved in the secretion of acid into the resorption lacuna, a specialized acidic compartment for mineral bone matrix degradation $(12,13)$. To the best of our knowledge, this is the first reported case of osteopetrosis that carried the TCIRG1 and CLCN7 gene mutations.

In order to determine the effect of $C L C N 7$ c.1798-1G>T, a transcription experiment was subsequently conducted. The mutations that affect mRNA splicing have been revealed to account for a number of hereditary disorders (14-16). Three splicing mutations of CLCN7 have been reported in osteopetrosis thus far, including c.916+57A >T, c.1617+6_1617+7delTG and c. $2250+1 \mathrm{G}>\mathrm{A}(9,17)$. The splicing mutation, CLCN7 c.1798-1G>A, reported in the present study has eradicated the invariant $\mathrm{G}$ of the AG splice acceptor site of intron 19. 
Table II. PCR primers used for amplification of $C L C N 7$ gene.

\begin{tabular}{|c|c|c|}
\hline Primer & Sequence (5' to $3^{\prime}$ ) & Size (bp) \\
\hline Promoter F (rs960467) & GGAAGCCTCCACTCCGACCC & 475 \\
\hline Promoter R (rs960467) & GTGATGAGCGACGGCGACCA & \\
\hline Exon1 F & CGTTGCAGGTCACATGGTC & 470 \\
\hline Exon1 R & GCCTCCGAAGACTCCAGAC & \\
\hline Exon2 F & CGGATCAGTTCTGCTTCCAG & 511 \\
\hline Exon2 R & CATGCTGTCACTGCTGTCCT & \\
\hline Exon3+Exon4 F & TGCTGGGATTGTAGGTGTCA & 629 \\
\hline Exon3+Exon4 R & GAGCAGCCTTCTTGGTTACG & \\
\hline Exon5+Exon6 F & CACACTGGGCCCTTCATAAT & 810 \\
\hline Exon5+Exon6 R & TCTGCTCCTCCTGAGGTTGT & \\
\hline Exon7 F & GTGTCTGCTGCTCTCCTCAG & 243 \\
\hline Exon7 R & GCTCCTGAACCAGCAAAGAG & \\
\hline Exon8+Exon9 F (VNTR in intron 8) & GCTTGGCTGCTGTTTAGCTC & 764 \\
\hline Exon8+Exon9 R (VNTR in intron 8) & AAGCCCATCTCCCTGAGTG & \\
\hline Exon10+Exon11 F & GTGCTGACCCTGCTGTCTCT & 797 \\
\hline Exon10+Exon11 R & AGGACCAAGGCCTGACAGA & \\
\hline Exon12 F & CACTGGCAAGTCCAGAGAGG & 559 \\
\hline Exon12 R & GCAGCAACTGTGTGACATCC & \\
\hline Exon13 F & CCAGTGTGTTTCTCCCCTGT & 443 \\
\hline Exon13 R & CTGTGGTTTTTGCCAACAGA & \\
\hline Exon14 F & ATTGCTCTGCTGGACACCTT & 551 \\
\hline Exon14 R & GCAGGGCCTCACTTCCTAC & \\
\hline Exon15 F (rs12926089, rs12926669) & CAGTGTCCTCCATCAGGGACT & 401 \\
\hline Exon15 R (rs12926089, rs12926669) & CTCTGAGATCTGGGTGGACAG & \\
\hline Exon16 F & CTCCCAACGTGTGCTCTCTC & 306 \\
\hline Exon16 R & ATCCTCCTGCCTTGGTCTCT & \\
\hline Exon17 F & TGAGAACAGGGAGCCTTCTG & 432 \\
\hline Exon17 R & AGGTGCGACACTTTTGTCCT & \\
\hline Exon18+Exon19 F & GGTGACTGTGCCCTCTGC & 730 \\
\hline Exon18+Exon19 R & CCCAGAAACCCTGAGCCTAC & \\
\hline Exon $20+$ Exon $21 \mathrm{~F}$ & CTGTGAGCCTCCAAACAGC & 717 \\
\hline Exon20+Exon21 R & GTCCACACAGCCCTCCAT & \\
\hline Exon22+Exon23 F & AGGCTGGTGTGAGCAGGTAG & 638 \\
\hline Exon22+Exon23 R & GCCCCTTGACTTCAGCTCTA & \\
\hline Exon24+Exon25 F & CTGAAGTCAAGGGGCTGAGG & 806 \\
\hline Exon24+Exon25 R & AGACCACTGCCCACAACAG & \\
\hline
\end{tabular}

PCR, polymerase chain reaction; CLCN7, chloride channel 7 gene; F, forward; R, reverse.

The most common consequence of splicing mutations is exon skipping, followed by the activation of aberrant splice sites and intron retention $(18,19)$. In the present study, two aberrant patterns of transcripts were detected with different proportions. The most common transcript was the skipping of exon 20 (c.1798_1883), which was predicted to cause a frameshift and a premature termination codon (p.Leu601GlyfsX13). The least common was the activation of aberrant splice sites (c.1798_1821 deletion), which was predicted to cause the in-frame deletion of eight amino acid residues (p.Gly600_Gln607del). Notably, aberrant splicing is predicted to affect only the $\mathrm{C}$-terminal cytosolic portion of the $\mathrm{ClCN} 7$ protein and not its transmembrane portion.
Mutations in the CLCN7 gene have been demonstrated to be involved in the pathogenesis of various forms of osteopetrosis since 2001 (12). Heterozygous mutations in CLCN7 may lead to ADO II, which is associated with less severe clinical features and late onset. To further investigate whether the CLCN7 c.1798-1G>T mutation was pathogenic, a thorough biochemical and radiological examination was performed on the 29-year-old mother who harbored the heterozygous CLCN7 splicing and TCIRG1 p.Tyr303X mutations. However, no abnormal clinical, biochemical or radiological manifestations were observed. Previous studies have reported several polymorphisms in the CLCN7 gene, including rs960467, 
Table III. Molecular analysis of the TCIRG1 and CLCN7 genes.

\begin{tabular}{|c|c|c|c|c|c|c|}
\hline \multirow[b]{2}{*}{ Subject } & \multirow{2}{*}{$\begin{array}{l}\text { Nucleotide change } \\
\text { of } T C I R G 1^{\text {a }}\end{array}$} & \multirow{2}{*}{$\begin{array}{l}\text { Nucleotide change } \\
\text { of } C L C N 7^{\mathrm{b}}\end{array}$} & \multicolumn{4}{|c|}{ Genotypes $^{c}$} \\
\hline & & & rs960467 & rs12926089 & rs12926669 & Intron 8 VNTR \\
\hline Patient & $\begin{array}{l}\text { c. } 909 \mathrm{C}>\mathrm{A} \\
\text { c. } 2008 \mathrm{C}>\mathrm{T}\end{array}$ & c. $1798-1 \mathrm{G}>\mathrm{T}$ & $\mathrm{G} / \mathrm{A}$ & $\mathrm{G}$ & $\mathrm{T}$ & Three repeat units \\
\hline Mother & c. $909 \mathrm{C}>\mathrm{A}$ & c. $1798-1 \mathrm{G}>\mathrm{T}$ & $\mathrm{G} / \mathrm{A}$ & $\mathrm{G}$ & $\mathrm{T}$ & Three repeat units \\
\hline Father & c. $2008 \mathrm{C}>\mathrm{T}$ & $-{ }^{\mathrm{d}}$ & $\mathrm{G}$ & $\mathrm{G}$ & $\mathrm{T}$ & Three repeat units \\
\hline
\end{tabular}

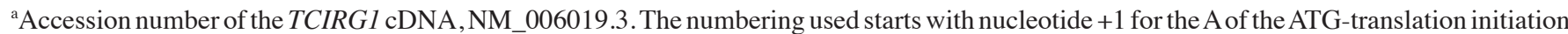
codon. ${ }^{\mathrm{b}}$ Accession number of the $C L C N 7$ cDNA, NM_001287.5. The numbering used starts with nucleotide +1 for the A of the ATG-translation initiation codon. ${ }^{\mathrm{r}} \mathrm{rs} 960467, C L C N 7$ NG_007567.1:g.4907G>A; rs12926089, CLCN7 NM_001287.5:c.1252G>A,NP_001278.1:p.Val418Met; rs12926669, CLCN7 NM_001287.5:c.1245T>C, NP_001278.1:p.Ile415=; Intron 8 VNTR, a 50-bp VNTR residing in intron 8 of $C L C N 7 .{ }^{\mathrm{d} N o}$ nucleotide change. VNTR, variable number tandem repeat.

A

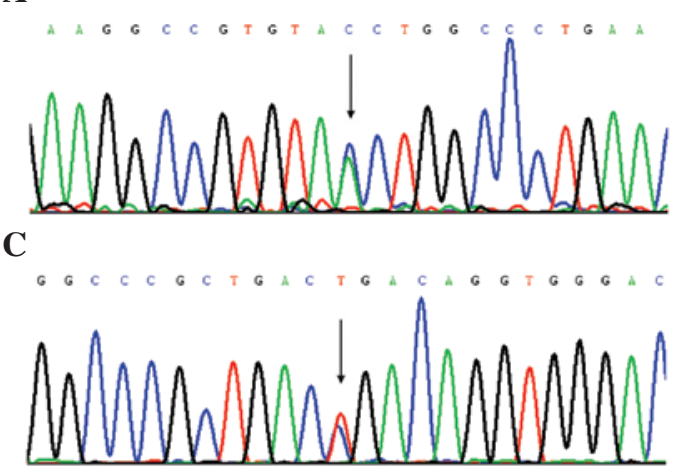

$\mathbf{E}$

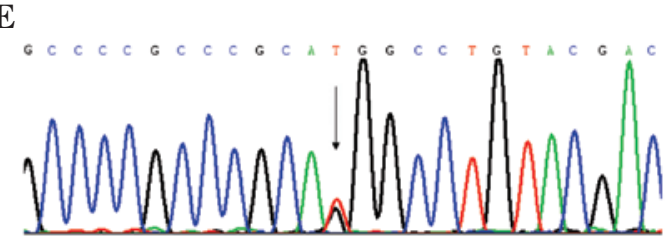

B

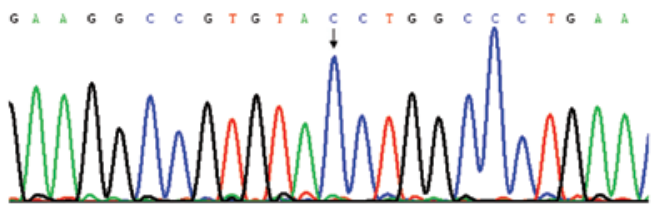

D

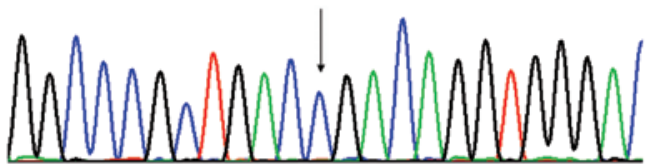

$\mathbf{F}$

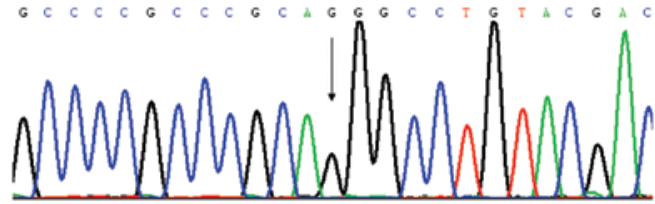

Figure 2. Molecular analysis of the TCIRG1 and CLCN7 genes. A genetic analysis showing (A) the TCIRG1 c.909C $>$ A (p.Y303X), (C) TCIRG1 c.2008C $>$ T (p.R670X) and (E) CLCN7 c.1798-1G>T mutations in the proband and (B, D , and F) the corresponding sequencing of the normal control. The arrow indicates the site of heterozygous mutation.

A

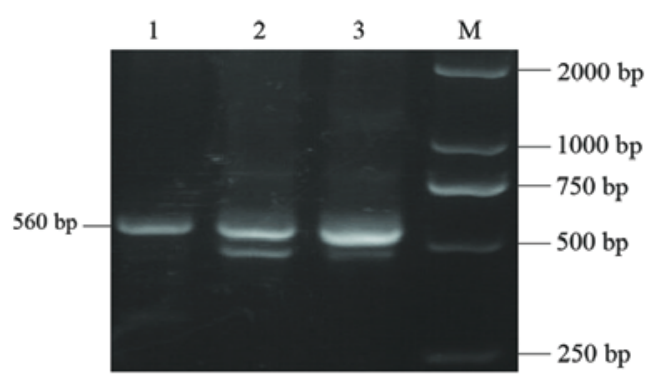

B Exon 19 Exon 21
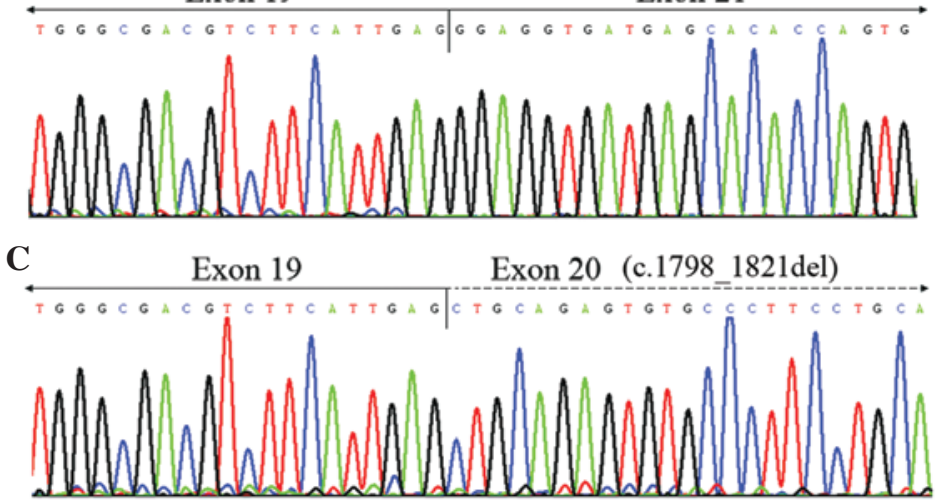

Figure 3. Transcription experiment of the $C L C N 7$ c.1798-1G $>$ T mutation (A) agarose-gel (1\%) electrophoresis of $C L C N 7$ RT-PCR products. RT-PCR performed on the RNA extracted from the father allowed detection of one band of the expected $560 \mathrm{bp}$, from exon 17 to exon 22 of the CLCN7 gene (lane 1). The same amplification conducted using RNA extracted from the mother (lane 2) and the proband (lane 3) resulted in two different PCR fragments. M, 2,000-bp DNA ladder. Cloning and sequencing of the pMD 18-T vectors containing CLCN7 RT-PCR products revealed two aberrant splicing forms, including (B) c.1798_1883 (exon 20) deletion and (C) c.1798_1821, the first 24 bp of the exon 20 deletion. The horizontal solid and dashed arrows indicate the areas of the two different exons. RT-PCR, reverse transcription polymerase chain reaction. 
rs12926089 (Val418Met) and rs12926669, and a VNTR in intron 8 , which were associated with the penetrance of the ADO phenotype and the variation in bone mineral density (20-22). To determine whether these polymorphisms were associated with the different severities of the osteopetrosis in the pedigree, the SNPs and the VNTR were genotyped. No difference was revealed between the patient and the mother. The CLCN7 c.1798-1G>T mutation appeared to be a non-pathogenic variation in the present case, although it was associated with aberrant splicing. However, completely excluding the pathogenicity of $C L C N 7$ c.1798-1G $>\mathrm{T}$ is inappropriate due to the incomplete penetrance of ADO II. The factors involved in the phenotypic variability remain unknown.

According to previous studies, the compound heterozygous nonsense mutations of TCIRG1 were enough to cause malignant osteopetrosis (5-7). Biallelic mutations in the TCIRG1 gene are well known to be responsible for ARO, and the absence of symptoms in the mother together with the characteristic ARO phenotype (early postnatal onset, generalized increased bone density and severe clinical course, including anemia, hypodontia and visual impairment) of the patient may indicate a diagnosis of classic TCIRG1-dependent ARO in this case.

In conclusion, a patient with $\mathrm{ARO}$ was studied. The compound heterozygous mutations, c.909C >A (p.Tyr303X) and c.2008C >T (p.Arg670X), in TCIRG1 and a heterozygous splicing mutation, c.1798-1G>T, in the CLCN7 gene were identified in the patient. Among the three mutations, TCIRG1 c.909C>A (p.Tyr303X) and $C L C N 7$ c.1798-1G>T were novel mutations. This study highlights that TCIRG1 and CLCN7 should be sequenced in order to gain a comprehensive molecular diagnosis of osteopetrosis.

\section{Acknowledgements}

The authors would like to thank all the members of the family for their participation in this study. This study was supported by the National Natural Science Foundation of China (grant nos. 81000207 and 81000346) and a foundation grant from Shanghai Science and Technology Commission for major issues (grant no. 11dz1950300).

\section{References}

1. Tolar J, Teitelbaum SL and Orchard PJ: Osteopetrosis. N Engl J Med 351: 2839-2849, 2004.

2. Stark Z and Savarirayan R: Osteopetrosis. Orphanet J Rare Dis 4: 5, 2009.

3. Loría-Cortés R, Quesada-Calvo E and Cordero-Chaverri C: Osteopetrosis in children: a report of 26 cases. J Pediatr 91: 43-47, 1977.

4. Bollerslev J and Andersen PE Jr: Radiological, biochemical and hereditary evidence of two types of autosomal dominant osteopetrosis. Bone 9: 7-13, 1988.

5. Frattini A, Orchard PJ, Sobacchi C, Giliani S, Abinun M, Mattsson JP, Keeling DJ, Andersson AK, Wallbrandt P, Zecca L, et al: Defects in TCIRG1 subunit of the vacuolar proton pump are responsible for a subset of human autosomal recessive osteopetrosis. Nat Genet 25: 343-346, 2000.
6. Kornak U, Schulz A, Friedrich W, Uhlhaas S, Kremens B, Voit T, Hasan C, Bode U, Jentsch TJ and Kubisch C: Mutations in the a3 subunit of the vacuolar $\mathrm{H}(+)$-ATPase cause infantile malignant osteopetrosis. Hum Mol Genet 9: 2059-2063, 2000.

7. Sobacchi C, Frattini A, Orchard P, Porras O, Tezcan I, Andolina M, Babul-Hirji R, Baric I, Canham N, Chitayat $\mathrm{D}$, et al: The mutational spectrum of human malignant autosomal recessive osteopetrosis. Hum Mol Genet 10: 1767-1773, 2001

8. Cleiren E, Bénichou O, Van Hul E, Gram J, Bollerslev J, Singer FR, Beaverson K, Aledo A, Whyte MP, Yoneyama T, et al: Albers-Schönberg disease (autosomal dominant osteopetrosis, type II) results from mutations in the $\mathrm{ClCN} 7$ chloride channel gene. Hum Mol Genet 10: 2861-2867, 2001.

9. Frattini A, Pangrazio A, Susani L, Sobacchi C, Mirolo M, Abinun M, Andolina M, Flanagan A, Horwitz EM, Mihci E, et al: Chloride channel ClCN7 mutations are responsible for severe recessive, dominant, and intermediate osteopetrosis. J Bone Miner Res 18: 1740-1747, 2003.

10. Del Fattore A, Peruzzi B, Rucci N, Recchia I, Cappariello A, Longo M, Fortunati D, Ballanti P, Iacobini M, Luciani M, et al: Clinical, genetic, and cellular analysis of 49 osteopetrotic patients: implications for diagnosis and treatment. J Med Genet 43: 315-325, 2006.

11. Supanchart $C$ and Kornak U: Ion channels and transporters in osteoclasts. Arch Biochem Biophys 473: 161-165, 2008.

12. Kornak U, Kasper D, Bösl MR, Kaiser E, Schweizer M, Schulz A, Friedrich W, Delling G and Jentsch TJ: Loss of the ClC-7 chloride channel leads to osteopetrosis in mice and man. Cell 104: 205-215, 2001.

13. Väänänen HK, Zhao H, Mulari M and Halleen JM: The cell biology of osteoclast function. J Cell Sci 113: 377-381, 2000.

14. Ars E, Serra E, García J, Kruyer H, Gaona A, Lázaro C and Estivill X: Mutations affecting mRNA splicing are the most common molecular defects in patients with neurofibromatosis type 1. Hum Mol Genet 9: 237-247, 2000.

15. López-Bigas N, Audit B, Ouzounis C, Parra G and Guigó R: Are splicing mutations the most frequent cause of hereditary disease? FEBS Lett 579: 1900-1903, 2005.

16. Yu T, Wang X, Ding Q, Fu Q, Dai J, Lu Y, Xi X and Wang H: Using a minigene approach to characterize a novel splice site mutation in human F7 gene causing inherited factor VII deficiency in a Chinese pedigree. Haemophilia 15: 1262-1266, 2009.

17. Pangrazio A, Pusch M, Caldana E, Frattini A, Lanino E, Tamhankar PM, Phadke S, Lopez AG, Orchard P, Mihci E, et al: Molecular and clinical heterogeneity in CLCN7 dependent osteopetrosis report of 20 novel mutations. Hum Mutat 31: E1071-E1080, 2010.

18. Krawczak M, Reiss J and Cooper DN: The mutational spectrum of single base-pair substitutions in mRNA splice junctions of human genes: causes and consequences. Hum Genet 90: 41-54, 1992.

19. Nakai K and Sakamoto H: Construction of a novel database containing aberrant splicing mutations of mammalian genes. Gene 141: 171-177, 1994.

20. Chu K, Koller DL, Snyder R, Fishburn T, Lai D, Waguespack SG, Foroud $\mathrm{T}$ and Econs MJ: Analysis of variation in expression of autosomal dominant osteopetrosis type 2: searching for modifier genes. Bone 37: 655-661, 2005.

21. Pettersson U, Albagha OM, Mirolo M, Taranta A, Frattini A, McGuigan FE, Vezzoni P, Teti A, van Hul W, Reid DM, Villa A and Ralston SH: Polymorphisms of the CLCN7 gene are associated with BMD in women. J Bone Miner Res 20: 1960-1967, 2005.

22. Kornak U, Ostertag A, Branger S, Benichou O and de Vernejoul MC: Polymorphisms in the CLCN7 gene modulate bone density in postmenopausal women and in patients with autosomal dominant osteopetrosis type II. J Clin Endocrinol Metab 91: 995-1000, 2006. 